\title{
Transcriptional regulation of chicken leukocyte cell-derived chemotaxin 2 in response to toll-like receptor 3 stimulation
}

\author{
Seokhyun Lee, ${ }^{1, a}$, Ra Ham Lee ${ }^{1, a}$, Sung-Jo Kim², Hak-Kyo Lee ${ }^{1}$, Chong-Sam Na ${ }^{1, *}$, and Ki-Duk Song ${ }^{1,3, *}$
}

\author{
* Corresponding Authors: \\ Chong-Sam Na \\ Tel: +82-63-270-2607, Fax: +82-63-270-2614, \\ E-mail: csna@jbnu.ac.kr \\ Ki-Duk Song \\ Tel: +82-63-219-5523, Fax: +82-63-270-5637 \\ E-mail: kiduk.song@jbnu.ac.kr
}

'Department of Animal Biotechnology, College of Agricultural and Life Sciences, Jeonbuk National University, Jeonju 54896, Korea

2 Department of Biotechnology, Hoseo University, Asan 31499, Korea

${ }^{3}$ The Animal Molecular Genetics and Breeding Center, Jeonbuk National University, Jeonju 54896, Korea

a These authors contributed equally to this work.

ORCID

Seokhyun Lee

https://orcid.org/0000-0001-9985-5380

Ra Ham Lee

https://orcid.org/0000-0002-4928-9517

Sung-Jo Kim

https://orcid.org/0000-0003-4590-3644

Hak-Kyo Lee

https://orcid.org/0000-0001-5387-4885

Chong-Sam Na

https://orcid.org/0000-0002-8979-5633

Ki-Duk Song

https://orcid.org/0000-0003-2827-0873

Submitted Mar 7, 2019; Revised May 3, 2019; Accepted Jun 20, 2019
Objective: Leukocyte cell-derived chemotaxin 2 (LECT2) is associated with several physiological processes including inflammation, tumorigenesis, and natural killer $\mathrm{T}$ cell generation. Chicken LECT2 (chLECT2) gene was originally identified as one of the differentially expressed genes in chicken kidney tissue, where the chickens were fed with different calcium doses. In this study, the molecular characteristics and gene expression of chLECT2 were analyzed under the stimulation of toll-like receptor 3 (TLR3) ligand to understand the involvement of chLECT2 expression in chicken metabolic disorders.

Methods: Amino acid sequence of LECT2 proteins from various species including fowl, fish, and mammal were retrieved from the Ensembl database and subjected to Insilco analyses. In addition, the time- and dose-dependent expression of chLECT2 was examined in DF-1 cells which were stimulated with polyinosinic:polycytidylic acid (poly [I:C]), a TLR3 ligand. Further, to explore the transcription factors required for the transcription of chLECT2, DF-1 cells were treated with poly (I:C) in the presence or absence of the nuclear factor $\mathrm{\kappa B}(\mathrm{NF \kappa B})$ and activated protein 1 (AP-1) inhibitors.

Results: The amino acid sequence prediction of chLECT2 protein revealed that along with duck LECT2 (duLECT2), it has unique signal peptide different from other vertebrate orthologs, and only chLECT2 and duLECT2 have an additional 157 and 161 amino acids on their carboxyl terminus, respectively. Phylogenetic analysis suggested that chLECT2 is evolved from a common ancestor along with the actinopterygii hence, more closely related than to the mammals. Our quantitative polymerase chain reaction results showed that, the expression of chLECT2 was up-regulated significantly in DF-1 cells under the stimulation of poly (I:C) $(\mathrm{p}<0.05)$. However, in the presence of NFKB or AP-1 inhibitors, the expression of chLECT2 is suppressed suggesting that both $\mathrm{NF \kappa B}$ and AP-1 transcription factors are required for the induction of chLECT2 expression.

Conclusion: The present results suggest that chLECT2 gene might be a target gene of TLR3 signaling. For the future, the expression pattern or molecular mechanism of chLECT2 under stimulation of other innate immune receptors shall be studied. The protein function of chLECT2 will be more clearly understood if further investigation about the mechanism of LECT2 in TLR pathways is conducted.

Keywords: Chicken; Leukocyte Cell-derived Chemotaxin 2 (LECT2); Gene Expression; Innate Immune Receptor Signaling

\section{INTRODUCTION}

Leukocyte cell-derived chemotaxin 2 (LECT2) is a multifunctional protein secreted from liver, and a chemotactic factor for neutrophils. Human LECT2 has a molecular mass of $\sim 16 \mathrm{kDa}$ [1], with three intramolecular disulfide bonds [2]. The amino acid sequence has been well conserved throughout evolution. Accumulating evidence indicates that, LECT2 is associated with several physiologic functions including regulation of liver regeneration 
[3], tumorigenesis [4,5], suppression of inflammatory arthritis and cytokine production [6,7] and hepatic natural killer $\mathrm{T}$ (NKT) cells generation [8]. It is reported that plasma LECT2 concentration of patients with bacterial sepsis is significantly lower than healthy humans and LECT2 expression is correlated with systemic inflammation [9]. In LECT2-knockout mice, hepatitis gets more severe due to the increased number of NKT cells and their cytokine ligands, and therefore, the homeostasis of NKT cells in the liver is negatively regulated by LECT2 $[8,10]$. It was also reported that LECT2 has antiinflammatory and tumor-suppressive actions in oncogenic $\beta$-catenin-induced liver tumorigenesis [5]. Human LECT2 is associated with inflammation and insulin resistance and the suppression of inflammatory and immune responses by LECT2 can inhibit tumorigenesis in liver [11,12].

In chicken, LECT2 was identified as mim-1 protein, P33, which is chemotactic for heterophils [13], and it also activates antimicrobial responses toward Salmonella enteritidis from chicken heterophils [13]. In Salmonella enteritidis infected chickens, the level of LECT2 protein was decreased in heterophils compared to macrophages without difference at RNA level $[14,15]$. In addition, the expression of duck LECT2 was significantly increased in liver and spleen by duck hepatitis virus type I infection [16], or the treatment of polyinosinicpolycytidylic acid (poly [I:C]), implying the LECT2 may play a role in the defense mechanism against viral infections in duck and be regulated by innate immune receptor signaling pathways, i.e., toll-like receptors 3 (TLR3).

The recognition of the microbial molecular patterns by TLRs trigger the intracellular signaling cascades where transcription factors, such as nuclear factor $\kappa \mathrm{B}(\mathrm{NF \kappa B})$ and activated protein 1 (AP-1) are activated for the transcriptional regulation of target genes [17]. In chicken, flagellin and lipopolysaccharide, which are bacterial products, activate both $\mathrm{NF \kappa B}$ and AP-1 signaling pathways; the engagements of TLRsTLR ligands lead to the degradation of the inhibitor of $\mathrm{NFKB}$

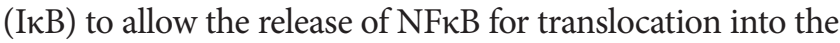
nucleus, and activate mitogen-activated protein kinase to phosphorylate AP-1, leading to the binding to the promoter of the target genes by activated NFKB and AP-1 $[18,19]$.

In this study, we evaluated transcriptional regulation of chicken LECT2 (chLECT2) in response to a TLR3 ligand engagements and explored signaling pathways responsible for the transcription of chLEC2 with the inhibitors of $\mathrm{NF \kappa B}$ and AP-1 in chicken DF-1 cells.

\section{MATERIALS AND METHODS}

\section{Bioinformatics analysis}

The LECT2 amino acid sequences of various species (chicken, duck, zebrafish, asian seabass, mouse, rat, pig, cow, human, and chimpanzee) were retrieved from the Ensembl database (http://www.ensembl.org/) (Table 1). The amino acid sequences were aligned with the ClustalW implemented in the BioEdit tool. The protein domains were predicted by using the SMART domain search program (http://smart.embl-heidelberg.de/). Phylogenetic analyses were performed with MEGA7 software [20].

\section{Cell culture}

The chicken DF-1 cell line was purchased from the American Tissue Culture Collection (CRL-12203, Manassas, VA, USA). DF-1 cells were cultured in Dulbecco's modified eagle medium, supplemented with $10 \%$ fetal bovine serum, $2 \mathrm{mM}$ L-glutamine and $100 \mathrm{U} / \mathrm{mL}$ each of penicillin and streptomycin (Thermo Scientific, Logan, UT, USA) at $37^{\circ} \mathrm{C}$ in a humidified atmosphere of $5 \% \mathrm{CO}_{2}$.

\section{Stimulation of DF- 1 cells by TLR3 ligand and inhibitors of NFKB and AP-1}

Poly (I:C) (TLR3 ligand) was purchased from InVivogen (San Diego, CA, USA) and dissolved in endotoxin and nuclease free water and kept at $-20^{\circ} \mathrm{C}$ until use. DF- 1 cells were treated at the concentrations of $0.1,1,5$, and $10 \mu \mathrm{g} / \mathrm{mL}$ for $24 \mathrm{~h}$. Also, to determine the time kinetics of mRNA expression, cells were treated for $1,3,6,12$, and $24 \mathrm{~h}$ at $10 \mu \mathrm{g} / \mathrm{mL}$ concentra-

Table 1. Ensembl and amino acid sequence ID in the leukocyte cell-derived chemotaxin 2 gene of various species

\begin{tabular}{llll}
\hline Species & Scientific name & \multicolumn{1}{c}{ Ensembl ID } & NCBI reference sequence ID \\
\hline Chicken & Gallus gallus & ENSGALG00000006323 & NM_205478.2 \\
Duck & Anas platyrhynchos & ENSAPLP00000004471 & KC250022.1 \\
Human & Homo sapiens & ENSG00000145826 & AB007546.1 \\
Chimpanzee & Pan troglodytes & ENSPTRG00000017263 & XM_517944.4 \\
Mouse & Mus musculus & ENSMUSG00000021539 & AB009687.1 \\
Rat & Rattus norvegicus & ENSRNOG00000012189 & NM_001108405.1 \\
Cow & Bos taurus & ENSBTAG00000001247 & AB001350.1 \\
Pig & Sus scrofa & ENSSSCG00000014315 & XM_021084783.1 \\
Horse & Equus caballus & ENSECAG00000008324 & XM_003362814.3 \\
Zebrafish & Danio rerio & ENSDARG00000086483 & DQ372604.1 \\
Asian Seabass & Lates calcarifer & N/A & KF717364.1 \\
\hline
\end{tabular}


tion. BAY 11-7085 and Tanshione IIA were purchased from Sigma-Aldrich (Louis, MO, USA). BAY 11-7085 (BAY) inhibits the IkBa phosphorylation, resulting in prevention of $\mathrm{NF \kappa B}$ activation, was used as NFkB inhibitor. Tanshinone IIA (Tan-II) may inhibit the binding of AP-1 to DNA in chicken heteophils [19]. DF-1 cells were pretreated with BAY 11-7085 $(5 \mu \mathrm{M})$ and Tan-II $(25 \mu \mathrm{M})$, for $3 \mathrm{~h}$, of which concentrations and time were determined from the preliminary experiment in this study, and then stimulated with poly (I:C) $(5 \mu \mathrm{g} / \mathrm{mL})$ for $3 \mathrm{~h}$.

\section{RNA extraction and cDNA preparation}

Trizol (Invitrogen, Carlsbad, CA, USA) was used to extract total RNA from chicken DF-1 cells. Total RNA was quantified by NanoDrop spectrophotometer (Thermo Fisher Scientific Inc., Waltham, MA, USA). After quantification, $2 \mu \mathrm{g}$ of total RNA was reverse-transcribed to synthesize cDNA using the using QuantiTect Reverse Transcription Kit (Toyobo, Osaka, Japan) according to the manufacturer's instructions.

\section{Quantitative real-time polymerase chain reaction analysis}

Quantitative real-time polymerase chain reaction (qRT-PCR) was conducted with a CFX-96 RT-PCR detection system (BioRad, Hercules, CA, USA) for the analysis of chLECT2 expression. The qRT-PCR conditions were as follows: an initial step at $94^{\circ} \mathrm{C}$ for $3 \mathrm{~min} ; 39$ cycles at $94^{\circ} \mathrm{C}$ for $10 \mathrm{~s}, 58^{\circ} \mathrm{C}$ for $30 \mathrm{~s}$, and $72^{\circ} \mathrm{C}$ for $30 \mathrm{~s}$; and a final step at $72^{\circ} \mathrm{C}$ for $10 \mathrm{~min}$. Dissociation was performed at $0.5^{\circ} \mathrm{C}$ increments from $55^{\circ} \mathrm{C}$ to $95^{\circ} \mathrm{C}$ for over $25 \mathrm{~min}$. The primer sequences of the chicken interleukin $1 \mathrm{~B}$ (chIL1B), chLECT2 and the glyceraldehyde 3-phos $\neg$ phate dehydrogenase (GAPDH) used in this study are shown in Table 2. All samples were measured in triplicates to ensure reproducibility, and $\mathrm{Ct}$ values were calculated by the $2^{-\Delta \Delta \mathrm{Ct}}$ method [21], to calculate the relative expression level of mRNA on each sample, which was expressed as a ratio relative to GAPDH expression.

\section{Statistical analysis}

Results were presented as means \pm standard deviation of trip-

Table 2. Quantitative polymerase chain reaction primers used for mRNA expression analysis

\begin{tabular}{llc}
\hline Target genes & \multicolumn{1}{c}{ Primers 5'-3' } & Accession no \\
\hline IL1B & F-GGATTC TGA GCA CAC CACAGT & XM_015297469.1 \\
& R- TCT GGT TGA TGT CGA AGA TGT C & \\
LECT2 & F-GAT ACG GCT GCG GCA ATT AC & NM_205478.2 \\
& R-GCC CTT GTG CTT TTC TCC TTT & \\
GAPDH & F-TGC TGC CCA GAA CAT CAT CC & NM_204305.1 \\
& R-ACG GCA GGT CAG GTCAACAA & \\
\hline
\end{tabular}

IL1B, interleukin 1B; LECT2, Leukocyte cell-derived chemotaxin 2; GAPDH, glyceraldehyde 3-phos $\neg$ phate dehydrogenase. licate independent experiments. Statistical significance was assessed using a Student's t-test. A value $\mathrm{p}<0.05$, as compared with the non-treated control, was considered statistically significant.

\section{RESULTS}

\section{Phylogenetic analysis of leukocyte cell-derived chemotaxin 2 sequences}

The amino acid sequence of chLECT2 was analyzed and compared with other species. The chLECT2 gene sequence was identified as a differentially expressed gene from chicken kidney RNA-seq study, where chickens were fed with different doses of calcium [22]. To verify and compare the chLECT2 amino acid sequences with other vertebrates, the amino acid sequences of LECT2 orthologs from fowl, fishes and mammal including duck, zebrafish, Asian seabass, mouse, rat, pig, cow, human and chimpanzee) were retrieved from ensemble database and aligned (Figure 1A). The analysis with SignalP v4.0 program (http://www.cbs.dtu.dk/) showed that chLECT2 and duck LECT2 (duLECT2) had the same signal peptide whose amino acid sequences starts at position 1 and ends at position 18. Besides, chLECT2 and duLECT2 had additional amino acids on carboxy terminus (157 for chicken and 161 amino acids for duck) compared to other species. The evolutionary relationships between chLECT2 and LECT2 orthologs were analyzed using phylogenetic tree (Figure 1). Phylogenetic analysis suggested that chLECT2 is evolved from a common ancestor with ducks. Also, it exhibited that LECT2 from fowls is relatively closely related that from fishes, while far from mammalian LECT2s.

\section{chLECT2 expression from DF-1 cells in response to TLR3 ligand stimulation}

To understand the expression patterns of this gene, the transcriptional profile of chLECT2 was investigated in chicken DF1 cells under the stimulation with TLR3 agonist, poly (I:C). To verify whether the expression of chLECT2 gene is regulated by TLR3 signaling, the expression of $\operatorname{chIL1B}$, which is known to be involved in TLR3 signaling, was also examined along with chLECT2. Stimulation with $10 \mu \mathrm{g} / \mathrm{mL}$ of poly (I:C) significantly upregulated both chLECT2 and ChIL1B expressions (Figure 2A, 2C). The levels of the chLECT2 and chIL1B were increased significantly from $1 \mathrm{~h}$ after stimulation with $10 \mu \mathrm{g} / \mathrm{mL}$ of poly (I:C), and reached to maximum at $6 \mathrm{~h}$ after stimulation, which lasted to $24 \mathrm{~h}$ (Figure 2B, 2D).

\section{Effects of transcription inhibitors on chLECT mRNA expression}

To determine whether transcription factors NFkB and AP-1 are responsible for chLECT2 transcription, DF-1 cells were pre-treated with NFkB inhibitor or AP-1 inhibitor before 


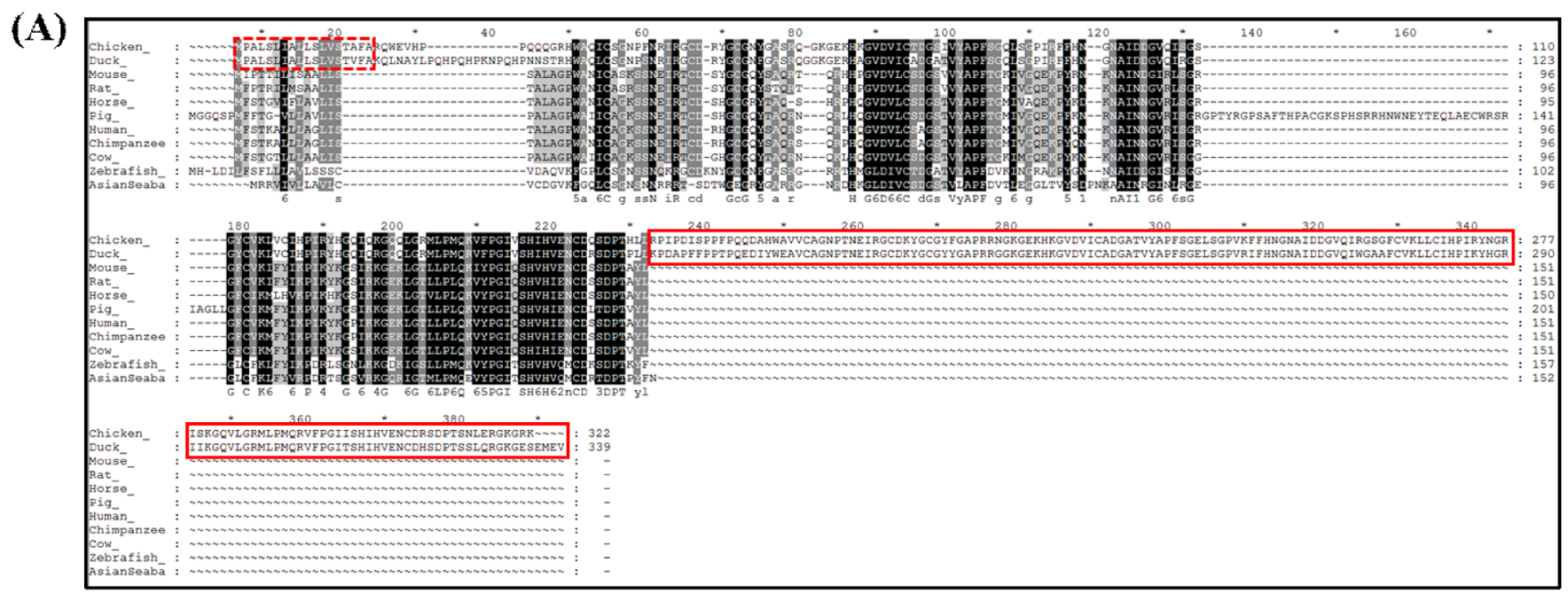

(B)

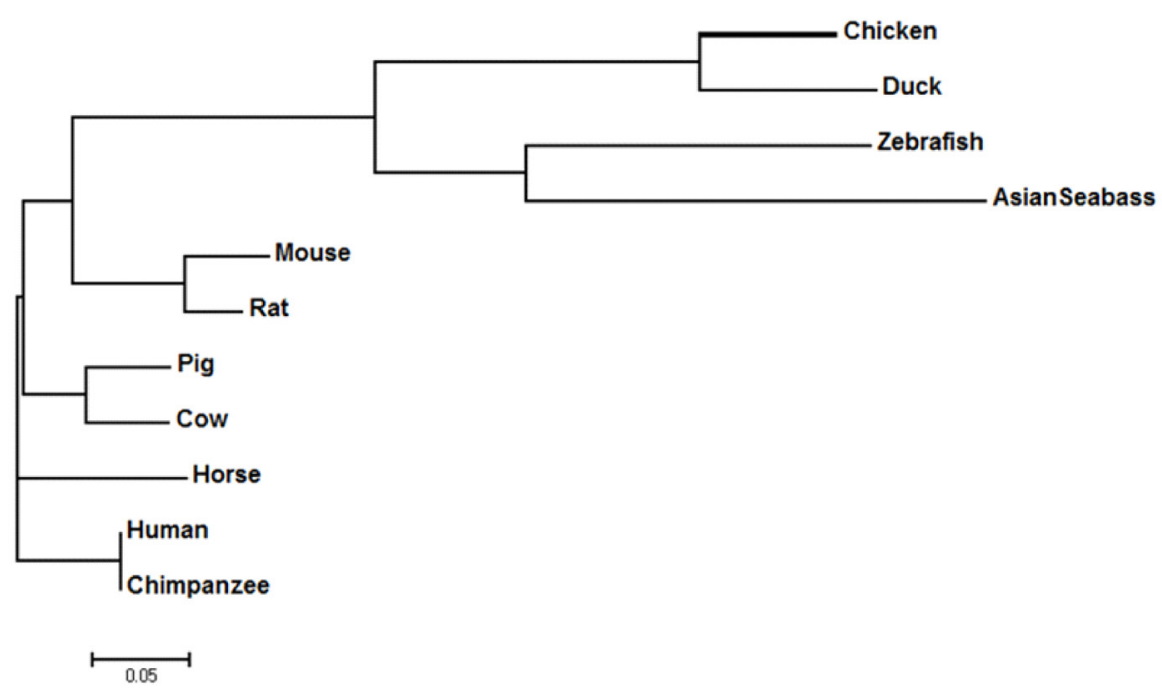

Figure 1. Analysis of LECT2 amino acid sequences. (A) Comparison of amino acid sequences of chicken LECT2 in various species (chicken, duck, zebrafish, Asian seabass, mouse, rat, pig, cow, horse, human, chimpanzee). Only chickens and ducks have the same signal peptide (dotted box) starting at position 1 and ending at position 18. In addition, the chicken and duck have unique 157 and 161 amino acids compared to other species (solid box). (B) Phylogenetic tree of LECT2 in various species. Phylogenetic analyses were performed with the amino acid sequence of each species by MEGA7 software method. ChLECT2 was clustered in the same clade with duck and fish group. The bar indicates 5\% amino acid divergence. LECT2, leukocyte cell-derived chemotaxin 2; chLECT2, chicken LECT2.

poly (I:C) stimulation (Figure 3). The expressions of both chLECT2 and $\operatorname{ChIL1B}$ were upregulated after treatment of 5 $\mu \mathrm{g} / \mathrm{mL}$ of poly (I:C) without the inhibitors. However, in the presence of transcriptional inhibitors, i.e., BAY or Tan-II, the expressions of chLECT2 and chIL1B were significantly suppressed compared to poly (I:C)-only treated control.

\section{DISCUSSION}

In mammals, LECT2 is known as a hepatokine mediating obesity with insulin resistance and associated with the inflammatory response $[23,24]$. It was also reported that LECT2 expression is increased in non-alcoholic fatty liver disease in association with metabolic syndromes such as abdominal obesity and lipid metabolism [12]. However, chLECT2 may play a different function compared to mammals. In previous report, we found the expression of LECT2 increased from the kidney of broiler chickens fed with excessive calcium, above that required for the growth of chickens including body weight gain and eggshell formation [22]. The excessively high calcium doses in feed cause reduced body weight gain and lead to stress-induced diseases, even though it caused the increased

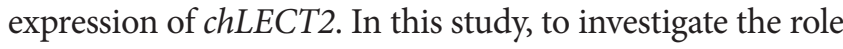
of chLECT2 in chicken immune response and metabolic disturbance, the expression patterns of chLECT2 under the stimulation of poly (I:C) revealed that the gene is involved in TLR3 signaling. Therefore, it is suggested that chLECT2 may be related to the innate immune system upon the viral infection. Interestingly, Bornelov et al [25] recently reported that chLECT2 expression in adipose tissue of layer hens is 
(A)

LECT2

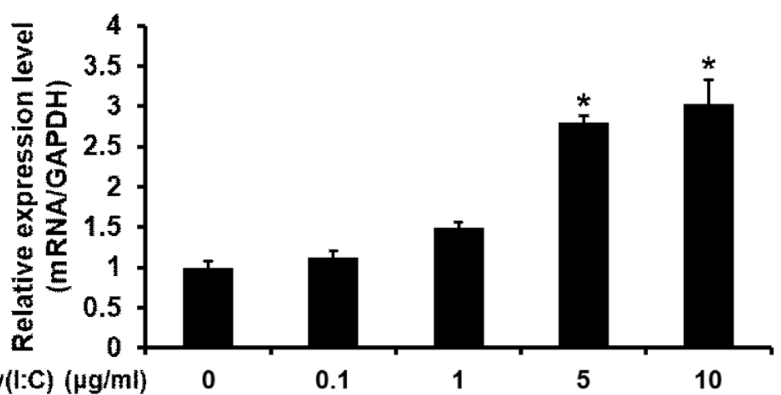

(C)

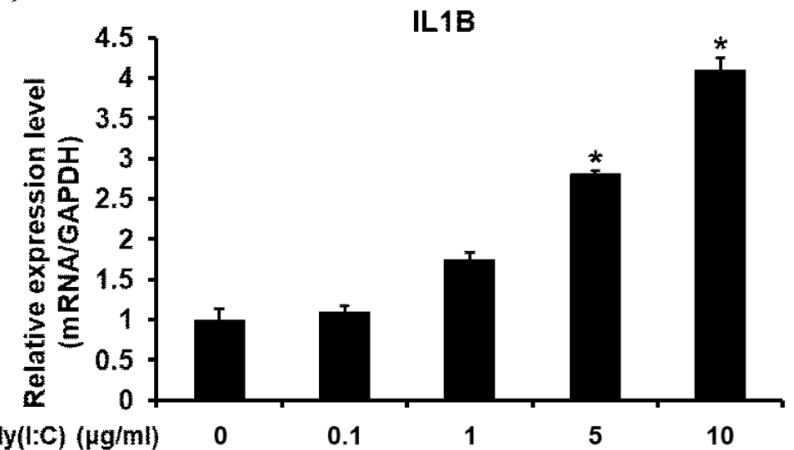

(B)

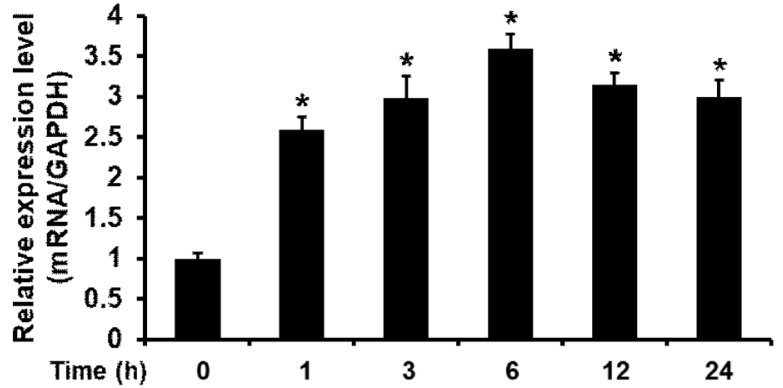

(D)

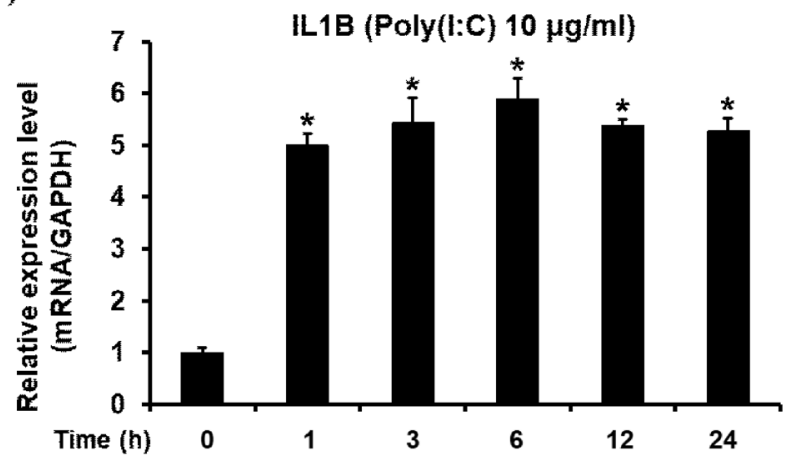

Figure 2. Analysis of expression levels of chLECT2 and chIL1B in the cultured chicken DF-1 fibroblast cells with the treatment of different concentration and time of poly (I:C). (A) and (C) Chicken DF-1 cells were treated with $0.1,1,5$, and $10 \mu \mathrm{g} / \mathrm{mL}$ concentrations of poly (I:C) for $24 \mathrm{~h}$, and the expressions of chLECT2 and chllL1B was analyzed by qRT-PCR. (B) and (D) Chicken DF-1 cells were treated with $10 \mu \mathrm{g} / \mathrm{mL}$ of poly (l:C) for 1, 3, 6, 12, and $24 \mathrm{~h}$, and then the expressional level of chLECT2 and chlL1B was analyzed by gRT-PCR. Each experiment was repeated for three times $(n=3)$. chLECT2, chicken leukocyte cell-derived chemotaxin 2; chlL1B, chicken interleukin 1B; poly (I:C), polyinosinic:polycytidylic acid; aRT-PCR, quantitative real-time polymerase chain reaction. Significant differences were determined by Tukey's test, and bars with * symbol on top are the cases that significant differences were not found $(p<0.05)$.

(A)

LECT2

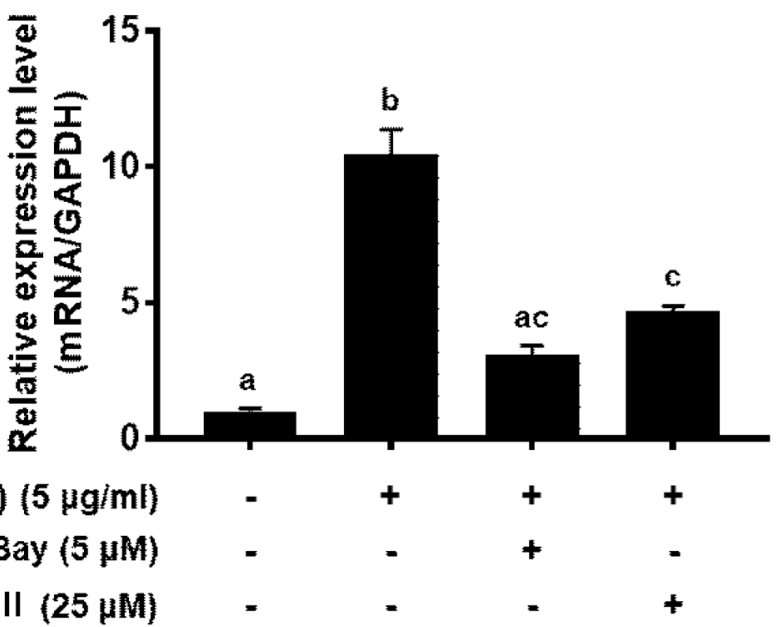

(B)

\section{IL1B}

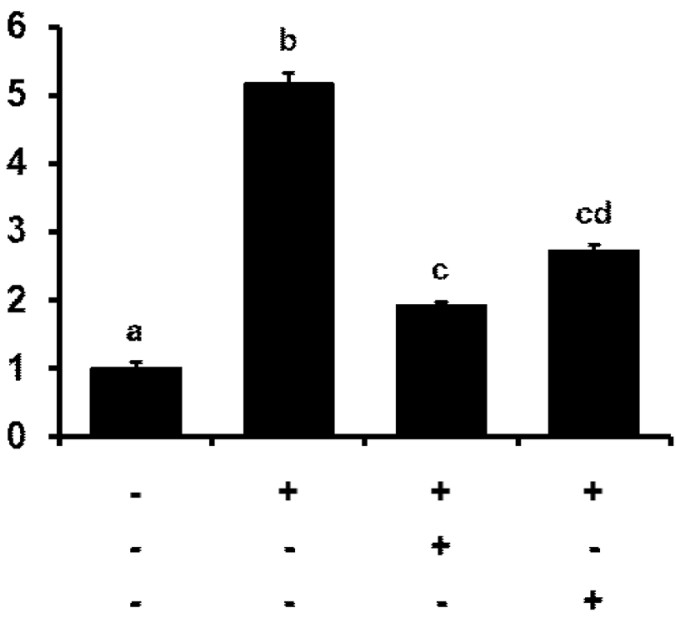

Figure 3. Effects of NFkB inhibitor (BAY11-7085; BAY) and AP-1 inhibitor (Tanshinone IIA; Tan-II) on the expressions of chLECT2 (A) and chIL1B (B) after stimulation with poly (I:C) in chicken DF-1 cells. The cells were treated with or without $5 \mu \mathrm{g} / \mathrm{mL}$ of poly $(\mathrm{l}: \mathrm{C})$ for $6 \mathrm{~h}$ in the presence or absence of $5 \mu \mathrm{M}$ of Bay or $25 \mu \mathrm{M}$ of Tan-II. Values are mean \pm standard error $(n=3)$. NFkB, nuclear factor $\kappa B$; AP-1, activated protein 1; chLECT2, chicken leukocyte cell-derived chemotaxin 2; chlL1B, chicken interleukin 1B; poly $(\mathrm{I}: \mathrm{C})$, polyinosinic:polycytidylic acid. Significant differences were determined by Tukey's test, and bars with the same letter $\left(\begin{array}{l}\text { a-d } \\ )\end{array}\right.$ on top are the cases that significant differences were not found (alpha<0.05). 
higher than broilers and suggested that the gene expression is related to the regulation of reproduction. Compared to this study, this means the function of LECT2 in chicken may vary tissue-specifically.

TLR3 plays a key role in initiating the immune response in chicken. As pathogens are detected by TLRs, the activation of transcription factors such as NF- $\kappa$ B and AP-1 is initiated. Afterwards, the transcriptional pathways stimulate the expression of pro-inflammatory cytokines including IL1B and type I interferon (IFN) and these downstream cytokines regulate the activity of immune-competent cells playing a role in innate immune defense systems. TLR3, as the receptor for viral dsRNA, triggers the antiviral immune pathway and induces the expression IFN which plays a critical role to antiviral activity.

Recently, Kamimura et al [26] reported that the transcription of the cytokine was induced by the transcriptional factors, NF- $\mathrm{KB}$ and AP-1 after stimulation of different TLR ligands in chicken vaginal cells. Also, they showed that the expression of $\operatorname{chIL} 1 B$ was suppressed by the inhibitors of the transcriptional factors, which are Bay for NF- $\kappa \mathrm{B}$, and Tan-II for AP-1. These results suggest that in chicken, poly (I:C) can stimulate the expression of chLECT2 and chIL1B through the activation of both transcriptional factors, NF- $\mathrm{KB}$ and AP-1. Therefore, as for the typical TLR3 signal pathway, the expression of chLECT 2 can be induced by NF- $\mathrm{kB}$ and AP- 1 under the stimulation of poly (I:C).

Besides mammals, several reports suggest that LECT2 plays important role in the inflammatory response and immune system in fish. The up-regulation of fish LECT2 expression under bacterial infection has been reported in many fishes, including zebrafish with Aeromonas salmonicida [27], croceine croaker with Vibrio alginolyticus [28], asian seabass with Vibrio harveyi [29], lamprey with Escherichia coli [30]. In Asian seabass and croceine croaker, the expression of LECT2 was significantly increased in liver and spleen with bacterial infection compared to healthy fish. As shown in Figure 1, the amino acid sequences of LECT2 orthologs have generally well conserved sequences but chLECT2 and duLECT2 have the same signal peptides and longer carboxy termini compared to other LECT2 orthologs. The phylogenetic analysis also revealed that chLECT2 is much closer to ducks and fishes than mammals. This might cause the similar but somewhat different function of chLECT2 compared to mammal orthologs.

Poly (I:C) is a prototypical class of pathogen-associated molecular patterns which are associated with pathogens. These molecules are recognized by TLRs and activate the innate immune system. Poly (I:C) is a stable synthetic dsRNA analogue which is generated during the viral replication, and is recognized by endosomally localized TLR3 ligand [31]. It is known that TLR3 prefers to recognize the synthetic poly (I:C) rather than virus-derived dsRNA, suggesting that TLR3 recognizes a unique dsRNA structure, different from other dsRNA-binding proteins [32]. The crucial role of TLR3 in poly (I:C) recognition was demonstrated by TLR3-knockout mice showing reduced inflammatory responses upon the treatment of poly (I:C) [33]. Many TLR3 effects rely on cells of the innate immune system that either express TLR3 or respond to inflammatory mediators that are produced upon TLR3 signaling.

In this study, we investigated the expression of chLECT2 from DF-1 cells in response to TLR3 ligand and identified that $\mathrm{NF \kappa B}$ and $\mathrm{AP}-1$ transcription factors are indispensable for TLR3-mediated expression of chLECT2. The genetic information and the expression patterns of chLECT2 revealed that chLECT2 is evolutionarily much closer to ducks or fishes than the mammal orthologs. Because LECT2 gene is known to be involved in inflammatory response, this report studied its biological role in chickens by investigating its expressional change under the treatment of TLR3 ligand and the inhibitors of transcriptional factors involved in TLR3 signaling. Also, the up-regulation of chLECT2 expression after the stimulation of poly (I:C) suggests that this gene might be related to viral infection in chickens, and the significantly lower expressional level of chLECT2 under the presence of transcriptional inhibitors, Bay and Tan-II verifies that the expression of this gene is involved in TLR3 signal pathway.

\section{CONCLUSION}

Exploring the expression profiles of chLECT2 and mechanisms by transcription factors of which activities are under TLR3 signals and regulate transcription of TLR3 target genes may provide fundamental knowledge of LECT2 in chicken. Further study may confirm the contribution of chLECT2 in the innate immune system against viral diseases and the chLECT2 gene is a promising candidate as a biomarker for infectious diseases in chickens.

\section{CONFLICT OF INTEREST}

We certify that there is no conflict of interest with any financial organization regarding the material discussed in the manuscript.

\section{ACKNOWLEDGMENTS}

The authors thank Dr. Vijayakumar Gosu for critical reading and editing of this manuscript. This work was supported by grants from the Next-Generation BioGreen 21 Program (No. PJ01324201, PJ01315101), Rural Development Administration, Republic of Korea. 


\section{REFERENCES}

1. Yamagoe S, Mizuno S, Suzuki K. Molecular cloning of human and bovine LECT2 having a neutrophil chemotactic activity and its specific expression in the liver. Biochim Biophys Acta 1998;1396:105-13. https://doi.org/10.1016/S0167-4781(97) 00181-4

2. Okumura A, Suzuki T, Dohmae N, et al. Identification and assignment of three disulfide bonds in mammalian leukocyte cell-derived chemotaxin 2 by matrix-assisted laser desorption/ ionization time-of-flight mass spectrometry. Biosci Trends 2009;3:139-43.

3. Sato $\mathrm{Y}$, Watanabe H, Kameyama H, et al. Changes in serum LECT 2 levels during the early period of liver regeneration after adult living related donor liver transplantation. Transplant Proc 2004;36:2357-8. https://doi.org/10.1016/j.transproceed. 2004.07.006

4. Ong HT, Tan PK, Wang SM, Hian Low DT, Ooi LL, Hui KM. The tumor suppressor function of LECT2 in human hepatocellular carcinoma makes it a potential therapeutic target. Cancer Gene Ther 2011;18:399-406. https://doi.org/10.1038/ cgt.2011.5

5. Anson M, Crain-Denoyelle AM, Baud V, et al. Oncogenic beta-catenin triggers an inflammatory response that determines the aggressiveness of hepatocellular carcinoma in mice. J Clin Invest 2012;122:586-99. https://doi.org/10.1172/JCI43937

6. Okumura A, Saito T, Otani I, et al. Suppressive role of leukocyte cell-derived chemotaxin 2 in mouse anti-type II collagen antibody-induced arthritis. Arthritis Rheum 2008;58:41321. https://doi.org/10.1002/art.23215

7. Dang MH, Kato H, Ueshiba H, et al. Possible role of LECT2 as an intrinsic regulatory factor in SEA-induced toxicity in d-galactosamine-sensitized mice. Clin Immunol 2010;137:31121. https://doi.org/10.1016/j.clim.2010.08.002

8. Saito T, Okumura A, Watanabe $\mathrm{H}$, et al. Increase in hepatic NKT cells in leukocyte cell-derived chemotaxin 2-deficient mice contributes to severe concanavalin A-induced hepatitis. J Immunol 2004;173:579-85. https://doi.org/10.4049/jimmunol. 173.1.579

9. Ando K, Kato H, Kotani T, Ozaki M, Arimura Y, Yagi J. Plasma leukocyte cell-derived chemotaxin 2 is associated with the severity of systemic inflammation in patients with sepsis. Microbiol Immunol 2012;56:708-18. https://doi.org/10.1111/ j.1348-0421.2012.00488.x

10. Ovejero C, Cavard C, Perianin A, et al. Identification of the leukocyte cell-derived chemotaxin 2 as a direct target gene of beta-catenin in the liver. Hepatology 2004;40:167-76. https:// doi.org/10.1002/hep.20286

11.Lu XJ, Chen J, Yu CH, et al. LECT2 protects mice against bacterial sepsis by activating macrophages via the CD209a receptor. J Exp Med 2013;210:5-13. https://doi.org/10.1084/ jem.20121466
12. Yoo HJ, Hwang SY, Choi JH, et al. Association of leukocyte cell-derived chemotaxin 2 (LECT2) with NAFLD, metabolic syndrome, and atherosclerosis. PLoS One 2017;12:e0174717. https://doi.org/10.1371/journal.pone.0174717

13. Bischoff KM, Pishko EJ, Genovese KJ, et al. Chicken mim-1 protein, P33, is a heterophil chemotactic factor present in Salmonella enteritidis immune lymphokine. J Food Prot 2001;64:1503-9. https://doi.org/10.4315/0362-028x-64.10. 1503

14. Sekelova Z, Stepanova H, Polansky O, et al. Differential protein expression in chicken macrophages and heterophils in vivo following infection with Salmonella Enteritidis. Vet Res 2017; 48:35. https://doi.org/10.1186/s13567-017-0439-0

15. Sekelova Z, Polansky O, Stepanova H, et al. Different roles of CD4, CD8 and gammadelta T-lymphocytes in naive and vaccinated chickens during Salmonella Enteritidis infection. Proteomics 2017;17:1700073. https://doi.org/10.1002/pmic. 201700073

16.Xu Q, Chen Y, Tong YY, et al. Identification and expression analysis of the leukocyte cell-derived chemotaxin-2 (LECT2) gene in duck (Anas platyrhynchos). Gene 2014;533:280-5. https://doi.org/10.1016/j.gene.2013.09.047

17. Kawai T, Akira S. The role of pattern-recognition receptors in innate immunity: update on Toll-like receptors. Nat Immunol 2010;11:373-84. https://doi.org/10.1038/ni.1863

18. Kogut MH, Genovese KJ, He H. Flagellin and lipopolysaccharide stimulate the MEK-ERK signaling pathway in chicken heterophils through differential activation of the small GTPases, Ras and Rap1. Mol Immunol 2007;44:1729-36. https://doi. org/10.1016/j.molimm.2006.07.292

19. Kogut MH, Genovese KJ, He H, Kaiser P. Flagellin and lipopolysaccharide up-regulation of IL- 6 and CXCLi2 gene expression in chicken heterophils is mediated by ERK1/2-dependent activation of AP-1 and NF-kappaB signaling pathways. Innate Immun 2008;14:213-22. https://doi.org/10.1177/175342 5908094416

20.Kumar S, Stecher G, Tamura K. MEGA7: Molecular Evolutionary genetics analysis version 7.0 for bigger datasets. Mol Biol Evol 2016;33:1870-4. https://doi.org/10.1093/molbev/ msw054

21.Livak KJ, Schmittgen TD. Analysis of relative gene expression data using real-time quantitative PCR and the 2(-Delta Delta C(T)) method. Methods 2001;25:402-8. https://doi.org/10. 1006/meth.2001.1262

22. Park W, Rengaraj D, Kil DY, Kim H, Lee HK, Song KD. RNAseq analysis of the kidneys of broiler chickens fed diets containing different concentrations of calcium. Sci Rep 2017;7:11740. https://doi.org/10.1038/s41598-017-11379-7

23.Lan F, Misu H, Chikamoto K, et al. LECT2 functions as a hepatokine that links obesity to skeletal muscle insulin resistance. Diabetes 2014;63:1649-64. https://doi.org/10.2337/ db13-0728 
24. Hwang HJ, Jung TW, Hong HC, et al. LECT2 induces atherosclerotic inflammatory reaction via $\mathrm{CD} 209$ receptor-mediated JNK phosphorylation in human endothelial cells. Metabolism 2015;64:1175-82. https://doi.org/10.1016/j.metabol.2015.06. 001

25. Bornelov S, Seroussi E, Yosefi S, et al. Comparative omics and feeding manipulations in chicken indicate a shift of the endocrine role of visceral fat towards reproduction. BMC Genomics 2018;19:295. https://doi.org/10.1186/s12864-018-4675-0

26. Kamimura T, Isobe N, Yoshimura Y. Effects of inhibitors of transcription factors, nuclear factor-kappaB and activator protein 1 , on the expression of proinflammatory cytokines and chemokines induced by stimulation with Toll-like receptor ligands in hen vaginal cells. Poult Sci 2017;96:723-30. https:// doi.org/10.3382/ps/pew366

27.Lin B, Chen S, Cao Z, et al. Acute phase response in zebrafish upon Aeromonas salmonicida and Staphylococcus aureus infection: striking similarities and obvious differences with mammals. Mol Immunol 2007;44:295-301. https://doi.org/ 10.1016/j.molimm.2006.03.001

28.Li MY, Chen J, Shi YH. Molecular cloning of leucocyte cellderived chemotaxin-2 gene in croceine croaker (Pseudosciaena crocea). Fish Shellfish Immunol 2008;24:252-6. https:// doi.org/10.1016/j.fsi.2007.09.003

29. Fu GH, Bai ZY, Xia JH, et al. Characterization of the LECT2 gene and its associations with resistance to the big belly disease in Asian seabass. Fish Shellfish Immunol 2014;37:131-8. https:// doi.org/10.1016/j.fsi.2014.01.019

30.Wang Z, Lu J, Li C, Li Q, Pang Y. Characterization of the LECT2 gene and its protective effects against microbial infection via large lymphocytes in Lampetra japonica. Dev Comp Immunol 2018;79:75-85. https://doi.org/10.1016/j.dci.2017.09.018

31. Weber F, Wagner V, Rasmussen SB, Hartmann R, Paludan SR. Double-stranded RNA is produced by positive-strand RNA viruses and DNA viruses but not in detectable amounts by negative-strand RNA viruses. J Virol 2006;80:5059-64. https://doi.org/10.1128/JVI.80.10.5059-5064.2006

32. Okahira S, Nishikawa F, Nishikawa S, Akazawa T, Seya T, Matsumoto M. Interferon-beta induction through toll-like receptor 3 depends on double-stranded RNA structure. DNA Cell Biol 2005;24:614-23. https://doi.org/10.1089/dna.2005. 24.614

33. Alexopoulou L, Holt AC, Medzhitov R, Flavell RA. Recognition of double-stranded RNA and activation of NF-kappaB by Toll-like receptor 3. Nature 2001;413:732-8. https://doi. org/10.1038/35099560 